\title{
Subacute hypersensitivity pneumonitis in an HIV infected patient receiving antiretroviral therapy
}

\author{
Alison M Morris, Stephen Nishimura, Laurence Huang
}

Department of Pulmonary and Critical Care Medicine, San Francisco General Hospital, University of California, San Francisco, California 94110, USA A $M$ Morris

L Huang

Department of Pathology S Nishimura

Correspondence to: Dr A M Morris, 995 Potrero Avenue, Building 80 , Ward 84, San Francisco General Hospital, San Francisco, CA 94110, USA email:

amorris@sfaids.ucsf.edu

Received 2 August 1999 Returned to authors 15 September 1999 Revised manuscript received 27 October 1999 Accepted for publication 25 November 1999

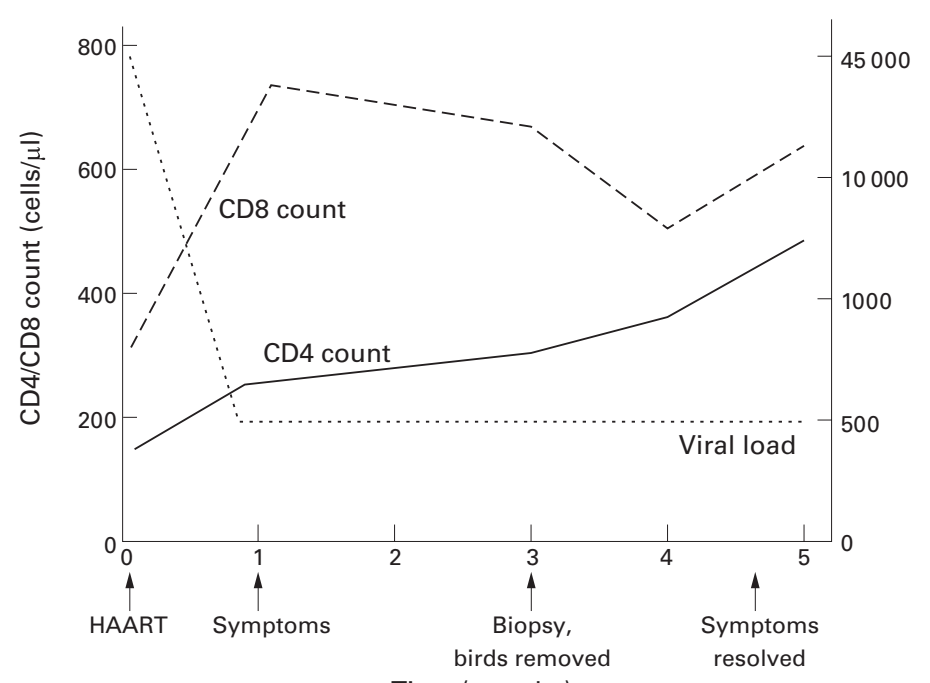

Time (months)

Figure 1 Time course of CD4 count, CD8 count, and viral load in relationship to clinical course.

\begin{abstract}
Abnormal pulmonary immune response to various antigens can lead to hypersensitivity pneumonitis. This disease has not previously been reported in HIV infected patients. This case report describes an HIV infected woman who developed subacute hypersensitivity pneumonitis in response to bird exposure. The disease manifested itself only after the patient experienced an improvement in her CD4 positive $T$ lymphocyte count secondary to antiretroviral therapy. This case emphasises the need to consider non-HIV associated diseases in patients with HIV and suggests that diseases in which host immune response plays an essential role in pathogenesis may become more prevalent in HIV infected patients receiving effective antiretroviral therapy.

(Thorax 2000;55:625-627)
\end{abstract}

Keywords: hypersensitivity pneumonitis; HIV; antiretroviral therapy

Hypersensitivity pneumonitis results from immunologically mediated lung damage provoked by antigen exposure. Common antigens include bird and animal proteins, chemicals, drugs, and fungi. ${ }^{1}$ Although the pathogenesis of hypersensitivity pneumonitis is incompletely understood, lymphocytes appear to play an important part. Bronchoalveolar lavage (BAL) fluid from patients with hypersensitivity pneumonitis contains increased numbers of lymphocytes, with relatively larger increases in (1)
CD8 positive $\mathrm{T}$ lymphocytes than in $\mathrm{CD} 4$ positive lymphocytes. ${ }^{23}$ Because the absolute numbers of CD4 and CD8 lymphocytes decrease along with the CD4 cell response to antigens in patients with advanced HIV infection, hypersensitivity pneumonitis should be an infrequent disease in this population. In fact, the literature contains no cases of HIV infected patients with hypersensitivity pneumonitis. However, many patients who receive highly active antiretroviral therapy (HAART) are experiencing improvement in their immune status, ${ }^{4}$ raising the possibility that diseases such as hypersensitivity pneumonitis, where host immune response plays an essential role in disease pathogenesis, may become more common. We report the case of a woman with AIDS who developed hypersensitivity pneumonitis after a significant improvement in her immune function due to HAART.

\section{Case report}

A 32 year old woman with AIDS and a nadir CD4 lymphocyte count of 86 cells/ $\mu$ l presented with three months of gradually increasing dyspnoea and non-productive cough. She denied fevers or chest pain, but had lost seven pounds in weight. She was a non-smoker and had a previous history of injection drug use, with none for the past three years. The patient was taking daily trimethoprim-sulfamethoxazole for Pneumocystis carinii pneumonia (PCP) prophylaxis. Her antiretroviral regimen consisted of abacavir (Ziagen; GlaxoWellcome, North Carolina, USA), nevirapine (Viramune; Roxane, Columbus, Ohio, USA), saquinavir (Invirase; Roche, Nutley, New Jersey, USA), and nelfinavir (Viracept; Agouron, LaJolla, California, USA). At the time of initiation of this regimen, the patient's CD4 count was $147 \mathrm{cells} / \mu \mathrm{l}$ and her viral load was 43800 copies/ml (fig 1). One month later her CD4 count had risen to 220 cells $/ \mu$ l, her viral load had become undetectable (less than 500 copies $/ \mathrm{ml}$ ), and her CD8 cell count was $728 \mathrm{cells} / \mu \mathrm{l}$. At this time she began experiencing mild shortness of breath. She did not present for evaluation until two months later when her CD 4 count was 273 cells/ $\mu$ l. Of note, the patient had owned lovebirds (Agapornis species) for two years and had acquired a button quail (Turnix species) and diamond dove (Geopelia species) four months prior to presentation.

Physical examination was unremarkable and her chest radiograph was normal. Oxygen saturation was $98 \%$ at rest, but decreased to $94 \%$ on exertion. Pulmonary function tests were remarkable for a transfer factor of $65 \%$ 


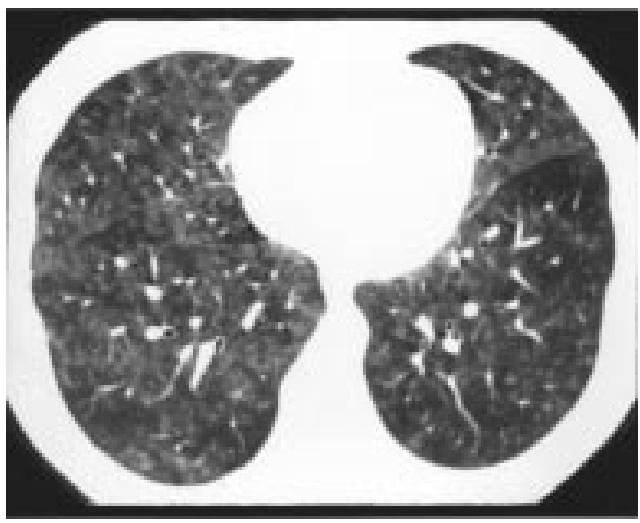

Figure 2 HRCT scan of the chest showing ill defined areas of ground glass opacities and multiple centrilobular nodules that become confluent in some areas.

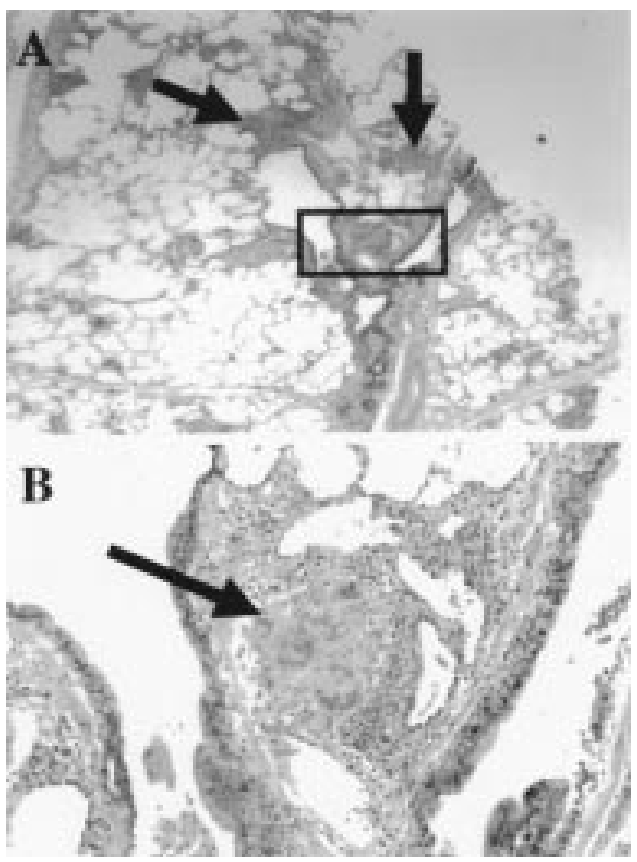

Figure 3 (A) Low power photomicrograph of a representative section of lung parenchyma obtained from an open lung biopsy specimen showing interstitial inflammation (arrows) extending out from a terminal bronchiole. (B) High power photomicrograph of the boxed region in A showing a non-necrotising granuloma with giant cells (arrows) adjacent to a bronchiole with a cellular lymphocytic bronchiolitis.

predicted, corrected for haemoglobin. Because of a suspicion for PCP, the patient underwent sputum induction and bronchoalveolar lavage (BAL), both of which were negative for Pneumocystis. Her symptoms progressed and her oxygen saturation declined to $92 \%$ at rest so further evaluation was performed. A high resolution computed tomographic (HRCT) chest scan showed patchy areas of ground glass opacities with evidence of air trapping and small nodules (fig 2). Repeat BAL with transbronchial biopsy specimens was negative for Pneumocystis and other pathogens. The BAL fluid showed lymphocytosis with a CD4/ CD8 ratio of 0.7 , similar to the ratio in her peripheral blood. Serum precipitins to pigeon antigens were negative.

Thoracoscopic lung biopsy sections were taken, and histological examination revealed a

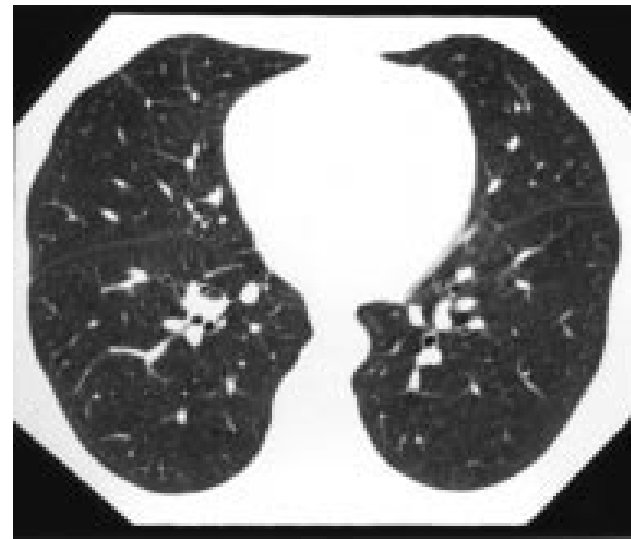

Figure 4 Repeat HRCT scan obtained six months after the end of the patient's exposure to birds. This image shows almost complete resolution of the ground glass opacities and nodules.

marked chronic interstitial inflammatory cell infiltrate with a cellular bronchiolitis of the medium and small airways (fig 3). Multiple small non-necrotising granulomas with giant cells were evenly distributed throughout the lung parenchyma. No organisms were seen on acid fast or fungal stains and no growth was observed in microbiological cultures. There was no evidence of injected silicates, established fibrosis, or honeycombing. Because the granulomas did not follow a bronchovascular pattern of distribution, the findings were felt to be more consistent with hypersensitivity pneumonitis than with sarcoidosis.

In this patient possible causes of hypersensitivity pneumonitis included her birds or antiretroviral medications. Because the patient had an excellent response to these medications, she was reluctant to discontinue them. It was therefore decided to remove her birds from the house. The patient did not receive steroids and the doses of her antiviral medications were not changed. Within several weeks her symptoms resolved completely. A repeat HRCT scan showed improvement of the ground glass opacities and nodules (fig 4). Repeat measurement of the transfer factor showed an improvement to $74 \%$ predicted.

\section{Discussion}

This patient's clinical history and pathology were consistent with subacute hypersensitivity pneumonitis which typically presents with the gradual onset of dyspnoea, cough, and weight loss. ${ }^{1}$ The chest radiograph may be normal or may have reticulonodular and ground glass infiltrates. The HRCT scan usually contains small nodules with areas of ground glass opacities. ${ }^{5}$ Air trapping may also be present. The patient's HRCT scan was highly suggestive of hypersensitivity pneumonitis, and the pathology was characteristic with findings of interstitial inflammation, bronchiolitis, and non-necrotising granulomas. ${ }^{6}$ The distribution and appearance of the granulomas were sufficient to distinguish the disease from sarcoidosis and mycobacterial or fungal disease.

BAL fluid lymphocyte subsets can be helpful in diagnosing hypersensitivity pneumonitis in 
the non-immunocompromised patient, but the interpretation of BAL lymphocyte subsets in the HIV infected patient is less straightforward. Studies have shown a BAL fluid lymphocytosis in HIV/AIDS patients with a CD4/CD8 ratio that generally reflects the ratio in the peripheral blood. ${ }^{7}$ These findings would be indistinguishable from those expected in hypersensitivity pneumonitis and would therefore not be useful in an HIV infected patient undergoing evaluation for hypersensitivity pneumonitis. ${ }^{3}$

This patient's sequence of development of hypersensitivity pneumonitis is intriguing. Her symptoms began shortly after she experienced a substantial increase in her CD4 lymphocyte count. The CD8 lymphocyte count has also been shown to increase when patients respond to HAART. ${ }^{9}$ Although difficult to prove conclusively, it is likely that the improvement in her immune function played some part in the development of hypersensitivity pneumonitis. This presentation is similar to cases of immune stimulation after initiation of HAART in the setting of infection with tuberculosis, Mycobacterium avium complex, and hepatitis C virus. ${ }^{10-14}$ In the present case the patient's immune system overreacted to an antigen instead of an infectious organism.

A recent report documented two cases of a sarcoid-like pulmonary illness that also occurred after immune reconstitution. ${ }^{15}$ These cases differed somewhat from the case presented here. One patient was asymptomatic and had chest radiograph abnormalities incidentally noted more than a year after the initiation of HAART. Treatment was not changed and the patient never became ill. The second patient developed respiratory symptoms shortly after beginning HAART. Treatment with interleukin-2 was discontinued, but antiretroviral agents were not changed. The patient's symptoms and pulmonary function gradually normalised. The pulmonary pathology in both these patients was consistent with sarcoidosis, not hypersensitivity pneumonitis as in our patient. Whether these various presentations are related solely to immune reconstitution or result from other factors cannot be fully determined; however, it is likely that the return of immune function played some part in the development of the hypersensitivity in our patient.

The advent of effective treatment for HIV infection has changed many aspects of the disease. Patients are surviving longer, but are also developing new and unusual complications. ${ }^{4}$ Given the dramatic immune response seen in patients treated with HAART, future observations of HIV infected patients with responses to HAART may show an increase in the prevalence of hypersensitivity pneumonitis and other immune mediated diseases.

1 Selman M. Hypersensitivity pneumonitis. In: Schwarz MI, King TE, eds. Interstitial lung disease. Hamilton: B C King TE, eds. Interstitial

2 Trentin L, Marcer G, Chilosi M, et al. Longitudinal study of alveolitis in hypersensitivity pneumonitis patients: an mmunologic evaluation. $\mathcal{F}$ Allergy Clin Immunol 1988;82: $577-85$

3 Wahlstrom J, Berlin M, Lundgren R, et al. Lung and blood T-cell receptor repertoire in extrinsic allergic alveolitis. Eur Respir F 1997;10:772-9.

4 Pallela FJ Jr, Delaney KM, Moorman AC, et al. Declining morbidity and mortality among patients with advanced human immunodeficiency virus infection. HIV Outpatient Study Investigators. N Engl f Med 1998;338:853-60.

5 Lynch DA, Rose CS, Way D, et al. Hypersensitivity pneumonitis: sensitivity of high-resolution CT scan in a pneumonitis: sensitivity of high-resolution CT

6 Coleman A, Colby TV. Histologic diagnosis of extrinsic Coleman A, Colby TV. Histologic diagnosis of extre
allergic alveolitis. Am $\mathcal{F}$ Surg Pathol 1988;12:514-8.

7 Guillon JM, Autran B, Denis M, et al. Human immunodeficiency virus-related lymphocytic alveolitis. Chest 1988; 94:18-22

8 Bofill M, Lipman M, McLaughlin JE, et al. Changes in lung lymphocyte populations reflect those seen in peripheral blood in HIV-1 positive individuals. Eur Respir F 1998;11: 548-53.

9 Lederman MM, Connick E, Landay A, et al. Immunologic responses associated with 12 weeks of combination antiretroviral therapy consisting of zidovudine, lamivudine, and roviral therapy consisting of zidovudine, lamivudine, and Infect Dis 1998;178:70-9.

10 Chien JW, Johnson JL. Paradoxical reactions in HIV and pulmonary TB. Chest 1998;114:933-6.

11 Dworkin MS, Fratkin MD. Mycobacterium avium complex lymph node abscess after use of highly active antiretroviral therapy in a patient with AIDS. Arch Intern Med 1998;158: 1828.

12 John M, Flexman J, French MA. Hepatitis C virusassociated hepatitis following treatment of HIV-infected patients with protease inhibitors: an immune restoration disease? AIDS 1998;12:2289-93.

13 Narita M, Ashkin D, Hollender ES, et al. Paradoxical worsening of tuberculosis following antiretroviral therapy in 2009-13.

14 Phillips P, Kwiatkowski MB, Copland M, et al. Mycobacterial lymphadenitis associated with the initiation of combination antiretroviral therapy. F Acquir Immune Defic Syndr Hum Retrovirol 1999;20:122-8.

5 Nacacche JM, Antoine M, Wislez M, et al. Sarcoid-like pulmonary disorder in human immunodeficiency virusinfected patients receiving antiretroviral therapy. $A m \mathcal{F}$ Respir Crit Care Med 1999;159:2009-13. 